\title{
BMJ Open Measuring changes in device use of a head-mounted low vision aid after personalised telerehabilitation: protocol for a feasibility study
}

Marie-Céline Lorenzini, ${ }^{\circledR 1,2,3}$ Walter Wittich $^{\circledR 1,2,3}$

To cite: Lorenzini M-C, Wittich W. Measuring changes in device use of a head-mounted low vision aid after personalised telerehabilitation: protocol for a feasibility study. BMJ Open 2019;9:e030149. doi:10.1136/ bmjopen-2019-030149

- Prepublication history for this paper is available online. To view these files, please visit the journal online (http://dx.doi. org/10.1136/bmjopen-2019030149).

Received 01 March 2019 Revised 30 August 2019 Accepted 05 September 2019

Check for updates

(C) Author(s) (or their employer(s)) 2019. Re-use permitted under CC BY-NC. No commercial re-use. See rights and permissions. Published by BMJ.

${ }^{1}$ School of Optometry, Université de Montréal, Montréal, Québec, Canada

${ }^{2}$ Centre de réadaptation Lethbridge-Layton-Mackay du CIUSSS du Centre-Ouest-del'île-de-Montréal, Centre de recherche interdisciplinaire en réadaptation du Montréal métropolitain, Montréal, Québec, Canada

${ }^{3}$ Institut Nazareth et LouisBraille du CISSS de la Montérégie-Centre, Centre de recherche interdisciplinaire en réadaptation du Montréal métropolitain, Montréal, Québec, Canada

\section{Correspondence to}

Marie-Céline Lorenzini;

marie-celine.Iorenzini@ umontreal.ca

\section{ABSTRACT}

Introduction A recent trend in low vision ( $\mathrm{LV}$ ) has been towards the use of portable head-mounted displays (HMDs) to enhance residual vision. The decision process around the (non-)use of such devices have been identified as multifactorial. Among important barriers identified in the context of magnifying LV aids were transportation issues and insufficient training. In recent years, telerehabilitation has become of growing interest in healthcare because it allows individuals to remain at home while receiving rehabilitation services. A recent pilot study indicated encouraging outcomes; however, very few applications of telerehabilitation for LV have been tested systematically. Methods and analysis To help guide evidence-based practice recommendations for this modality, we will carry out a feasibility study to assess the recruitment, retention, accessibility and acceptability of an eventual fully randomised trial of telerehabilitation for people with LV using HMDs. We will recruit 60 participants aged 18+ years among prospective eSight Eyewear owners, randomised 1:1 into two parallel groups. The active intervention will be the telerehabilitation operated by a LV therapist; the control arm will be the current selftraining standard provided by the device vendor. The primary feasibility outcome measures will be: time to recruit participants, loss to follow-up, accessibility and acceptability of the telerehabilitation (satisfaction of the users and LV therapist). Exploratory outcomes will be the impact of telerehabilitation on eSight Eyewear use behaviour (discontinuance rate), and validated measures of assistive-technology-related quality of life.

Ethics and dissemination The study was approved by the Ethics Review Board of the Centre de Recherche Interdisciplinaire en Réadaptation de Montréal métropolitain (CRIR\# 1286-1217). Dissemination is planned via local, national and international healthcare conferences and peer-reviewed journal publications.

\section{INTRODUCTION}

It is estimated that 314 million people are visually impaired worldwide, ${ }^{1}$ making this approximation a global concern that is likely to become more significant as average lifespans increase in many countries. Visual impairment includes blindness and low vision $(\mathrm{LV})$, and it is globally prevalent across the

\section{Strengths and limitations of this study}

- The randomised participant allocation, masking the researcher conducting the statistical analyses to group allocation, and the use of self-administered assessments will minimise selection, detection and experimenter bias.

- The telerehabilitation approach allows enrolment and participation across both rural and urban environments.

- Performance and social desirability bias might occur during participant assessment because their masking will not be possible.

- It will not always be possible to differentiate all possible reasons for discontinuance versus drop-out. This will specifically be the case for participants lost to follow-up without explanation.

- The absence of an in-office evaluation with objective visual function measures (eg, visual acuity, visual field, contrast sensitivity) and measures of functional vision (eg, related to daily living activities) are a limitation.

lifespan. ${ }^{2} \mathrm{LV}$ is defined as mild or moderate visual impairment that is not correctable with glasses, contact lenses or surgical intervention, and interferes with normal everyday functioning. ${ }^{3} \mathrm{LV}$ rehabilitation is the primary intervention for people with residual vision and has been shown to be effective, specifically in the context of magnification. ${ }^{4}$ The goal is to improve independence in activities of daily living and quality of life of people with reduced visual function by enhancing their remaining sight. ${ }^{4}$ One of the main methods of achieving such improvement is through the provision of and training in the use of $\mathrm{LV}$ aids (LVAs). Optical and electronic magnification devices, such as loupes, close-circuit television and telescopic systems are among the most common forms of intervention in a LV rehabilitation programme. ${ }^{5}$

With the massive evolution of new technologies, such as mobile and wearable devices, 
immersive systems (eg, virtual reality) have become more and more available for $\mathrm{LV}$ rehabilitation. ${ }^{6}$ A recent trend has been towards portable head-mounted displays (HMDs), providing hands-free magnification and contrast enhancements at all distances, using optoelectronics and real-time video technology. ${ }^{7}$ One of the first HMDs was the Low Vision Enhancement System that demonstrated its usefulness in adults ${ }^{8}$ and in children with $\mathrm{LV}$ by improving visual acuity, contrast sensitivity and control of ambient light. ${ }^{10}$ After considerable technological evolution, HMDs currently available such as the redesigned Jordy (Enhanced Vision Systems, Huntington Beach, California, USA), NuEyes Pro Smartglasses (NuEyes USA, Newport Beach, California, USA), CyberTimez Cyber Eyez (Cyber Timez, Winchester, Virginia, USA), Evergaze seeBOOST (Evergaze, LLC, Richardson, Texas, USA) and IrisVision (Visionize, LLC, Berkeley, California, USA), became smaller and lighter. Their performances have been compared with traditional optical LVAs (ie, magnifiers, closed circuit television, telescopic systems). ${ }^{11}$ The importance of training in the use of HMDs was demonstrated for distant and intermediate vision compared with traditional LVAs. ${ }^{12}$ One of the more recently developed HMDs, eSight Eyewear (eSight Corp., Toronto, Ontario, Canada), was designed to improve on previous devices by not only providing variable magnification, auto focus, contrast enhancement, hands-free use and portability but also offering digital image processing that allows the user to scan through a wide-field image. In 2018, a multicentre prospective trial investigated the effect of eSight Eyewear on 51 novice users for 3 months, demonstrating that activities of daily living and reading showed greatest benefit from device use. ${ }^{13}$

\section{Continuation and interruptions in the use of electronic assistive devices}

Despite the functional and evidence-based benefits of LVA use, ${ }^{4}{ }^{14}$ a scoping review documented high variability rates of device non-use for magnifying LVAs after their prescription, ranging from $2.3 \%$ to $50 \%$ ( $\mathrm{M}=25 \%$, $\mathrm{SD}=14 \%) .{ }^{15}$ In the context of eSight Eyewear, a cross-sectional study revealed that of 109 users, $17.4 \% \quad(n=19)$ had discontinued their use. ${ }^{16}$ The authors reported that reasons for non-use had been identified as multifactorial, involving the device, the user, the environment and the intervention. Among important barriers in the process of acquiring and incorporating magnifying LVAs were limited access to transportation to receive training with a device, insufficient training duration and frequency, and negative healthcare experiences including providers' poor knowledge of devices, absence of positive interactions between the patient/client and LV healthcare professionals as well as delays in obtaining an appointment. ${ }^{15}$ Regarding HMDs, a cross-sectional study concluded that device-related quality of life, absence of headaches and follow-up service satisfaction were important predictors of eSight Eyewear use. ${ }^{16}$ Transportation issues limited the ability of individuals to access follow-up interventions for their LVA training and skill reinforcement. ${ }^{17} 18$ This is particularly a challenge in the USA, given that two-thirds of the LV population do not or are not permitted to drive. ${ }^{19}$ Moreover, the paucity of $\mathrm{LV}$ rehabilitation providers exacerbates issues related to limited care access in rural areas of geographically dispersed countries, such as Australia, the USA and Canada. ${ }^{20} 21$ To overcome such barriers, innovative internet-based communication technology presents itself as a potential solution for rehabilitation services.

\section{Telerehabilitation: an alternative modality for $L V$ rehabilitation services}

In recent years, telerehabilitation has become of growing interest in healthcare because it allows individuals to remain at home while receiving rehabilitation services via information and communication technologies. ${ }^{22}$ Several advantages of telerehabilitation have been documented when compared with traditional in-office interventions, including overcoming transportation difficulties, optimising follow-up session scheduling and evaluation of the patients' environment. A systematic review on telerehabilitation across disabilities (but not including $\mathrm{LV}$ ) revealed that $71 \%$ of the interventions were successful, ${ }^{23}$ thereby supporting evidence-based practice recommendations towards implementation of remote online healthcare intervention.

In LV rehabilitation, visual aids using video systems (eg, smart phone, tablet) are used more and more by people with visual impairments and suggest that they could be used to provide telerehabilitation services. ${ }^{24}$ A Cochrane systematic review documented very few applications of telerehabilitation to $\mathrm{LV}$ and no published outcomes. ${ }^{25}$ Recently, a pilot study indicated encouraging outcomes confirming the feasibility and acceptability of training to optimise the use of handheld magnifiers in 10 patients with LV via telerehabilitation from their home. ${ }^{26}$ To help guide evidence-based practice recommendations for this modality, the main goal of the present protocol is to conduct a feasibility study using telerehabilitation operated by a LV therapist, compared with the current self-training standard provided by the device vendor, eSight Corporation. We expect to obtain evidence about feasibility (time to recruit participants, loss to follow-up, accessibility and acceptability) of administering several LV rehabilitation training sessions via telerehabilitation using an internet-based video platform to participants with LV using their eSight Eyewear at home. As secondary goals, it is planned to determine if personalised intervention through telerehabilitation can help to reduce discontinuance (or induce change in use) for HMDs and improve quality of life. In addition, other previously identified predictors of eSight Eyewear use, such as follow-up service satisfaction, will be explored. We hypothesise that personalised LV rehabilitation through telerehabilitation will be a feasible (accessible and acceptable) modality to train individuals with $\mathrm{LV}$ to use their eSight device in their environment, will reduce discontinuance and will improve quality of life. 


\section{METHODS AND ANALYSIS}

\section{Study design}

This feasibility study considered the Consolidated Standards of Reporting Trials guideline components, ${ }^{27}$ and is a parallel two-arm randomised study consisting of training individuals with LV in the use of eSight Eyewear when engaging in activities of daily living. The experimental intervention will entail a series of personalised LV telerehabilitation sessions with a LV rehabilitation specialist. The control intervention will consist of the conventional eSight self-training using their eSkills User Guide ${ }^{28}$ as well as optional access to standard support available through eSight Corporation staff. These staff members may either be normally sighted, or may be device users whose experience is based on their own visual impairment. The study will be based at the School of Optometry of the Université de Montréal, Montreal, Quebec, Canada, and follows the requirements of the Declaration of Helsinki for conducting research with human participants. ${ }^{29}$

\section{Telerehabilitation equipment}

In the case of personalised training by telerehabilitation, participants will benefit from distance training sessions delivered to their home via the Internet with a LV therapist at the School of Optometry of the Universite de Montréal. Each participant will be able to interact in real time, using a secure and password-protected connection, with the LV therapist using the REACTS telehealth platform, accessible from their computer or digital tablet at specific appointments (https://www.iitreacts.com).

We will include individuals with self-reported LV aged $18+$ years, who are able to communicate in English or French, have a tablet, desktop or laptop computer with internet access, are highly motivated to participate in the study, and recently ( $<1$ month) bought or are currently renting an eSight Eyewear device. Current device users who have owned their eSight device for $>1$ month (and have therefore completed the eSight eSkills User Guide), as well as those self-reporting other severe sensory impairments that may interfere with communication, will be excluded. The LV therapist will confirm whether participants are able to follow a 20 min phone conversation (ie, sufficient hearing and cognition to complete oral informed consent and protocol procedures by phone, based on her clinical experience), and will exclude them if comprehension and/or communication are challenged. Figure 1 summarises the design of the study; each of the study aspects is described in detail.

\section{Interventions}

Self-training standard provided by eSight. This intervention focuses on the technical aspect of using the eSight device, namely: 'how does eSight Eyewear work?'. All participants in the control group will receive the eSkills learning and training guide ${ }^{28}$ as usually provided when purchasing the device. This guide is a self-training programme that spans 1 hour per day for 1 month (30hours) to be performed self-guided at home, and divided into 4 weeks of exercises (see table 1). At the end of the training, the participants will continue to use the eSight Eyewear in their environment until the end of the study 5 months later (and beyond).

Personalised training through telerehabilitation. This intervention focuses on the functional aspect of using eSight, namely: 'How to achieve your activities of daily living with eSight Eyewear?'. The personalised training by telerehabilitation will be provided by the same and only LV therapist involved in the study and will consist of six 1 hour online training sessions within the first 2 weeks (6 hours), 12 additional hours of homework in parallel during the same 2 weeks and an additional 12 hours of homework in the following 2 weeks. The LV therapist and participants will share common work materials composed of exercises that they can easily refer to. The materials are composed of the eSkills learning and training guide ${ }^{28}$ and digitised exercises extracted from the VisExc-eccentric fixation programme ${ }^{30}$ (partially adapted from the McGill Low Vision Manual, ${ }^{31}$ sent by email for eccentric fixation training). The 6 sessions will be scheduled at the beginning of the study according to the participants' and the LV therapist's availabilities (see table 2). Individualised training in the use of the eSight Eyewear will be provided to each participant and tailored to the needs of each person, as would be the case in face-to-face clinical practice.

At each session, a 5 min break will be offered to participants when needed. Between each of these 6 sessions, participants are asked to continue to train themselves at home using the eSkills learning and training guide for $\sim 6$ hours per week (12 hours of homework). At the end of the 2 weeks (telerehabilitation intervention), the participants will continue their training for 2 weeks (12 hours of homework remaining) and continue using their eSight device at home, until the end of the study 5 months later (and beyond).

\section{Outcomes}

The primary outcome of this study is related to the feasibility of telerehabilitation in the context of eSight Eyewear users and encompasses several measures: (1) enrolment target (signed consent) within 10 months, (2) retention of participants until 6 months after randomisation, (3) accessibility of telerehabilitation training and (4) acceptability of telerehabilitation training.

\section{Primary outcomes}

Considering the feasibility aspect of the study, our enrolment target is 60 participants ( 30 renters and 30 buyers of eSight Eyewear) over 10 months. We will consider that a trial is feasible if we enrol $80 \%$ of this figure $(n=54)$ during this period and/or if $100 \%$ are enrolled over 12 months. We will record the number of eligible individuals declining to participate and why, and capture whether any participants dropped out of the study and why. Retention will be monitored by follow-up evaluations and through questionnaires. Accessibility of the training via telerehabilitation 
Recruitment

- Letter introducing the study sent by eSight to all the new eSight Eyewear owners

- New eSight Eyewear owners contact the research team

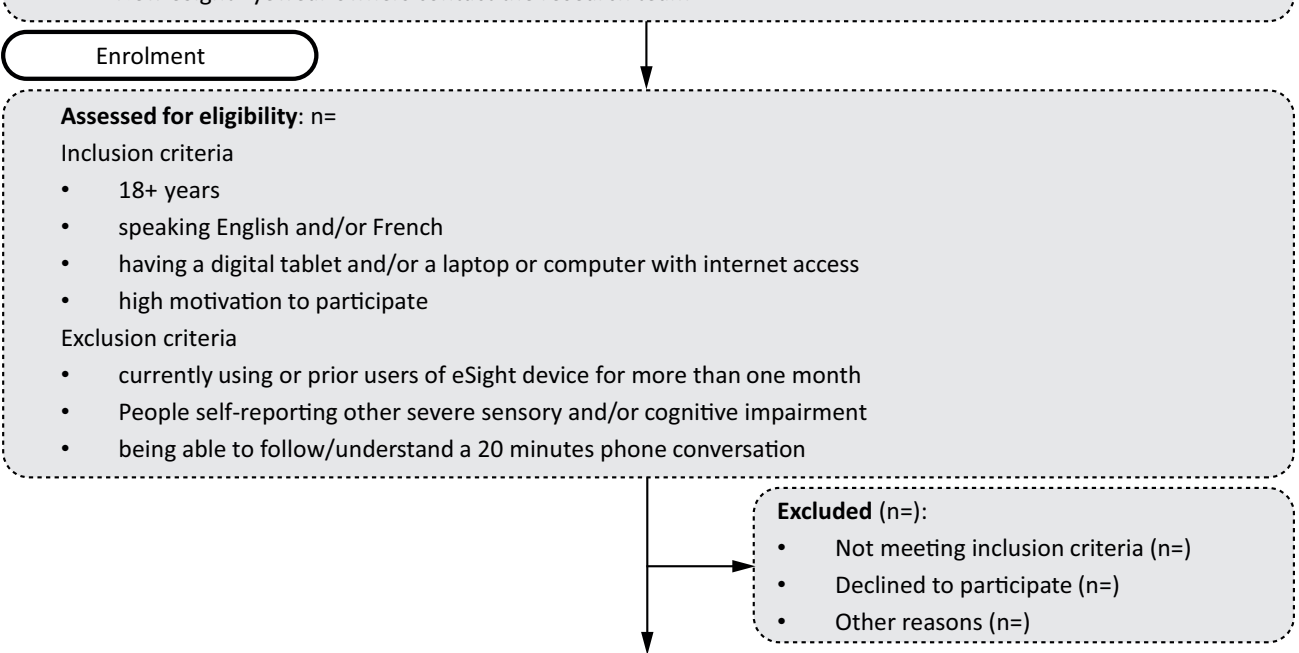

Randomized $(\mathrm{n}=)$

Two separate randomizations carried out separately at the Université de Montréal by a research assistant who is not involved in the study.

- Renter users $(n=)$

- Buyer users $(n=)$

\section{Baseline assessment}

- Demographic measures

- $\quad$ Device-related quality of life measures (the PIADS, the QUEST)

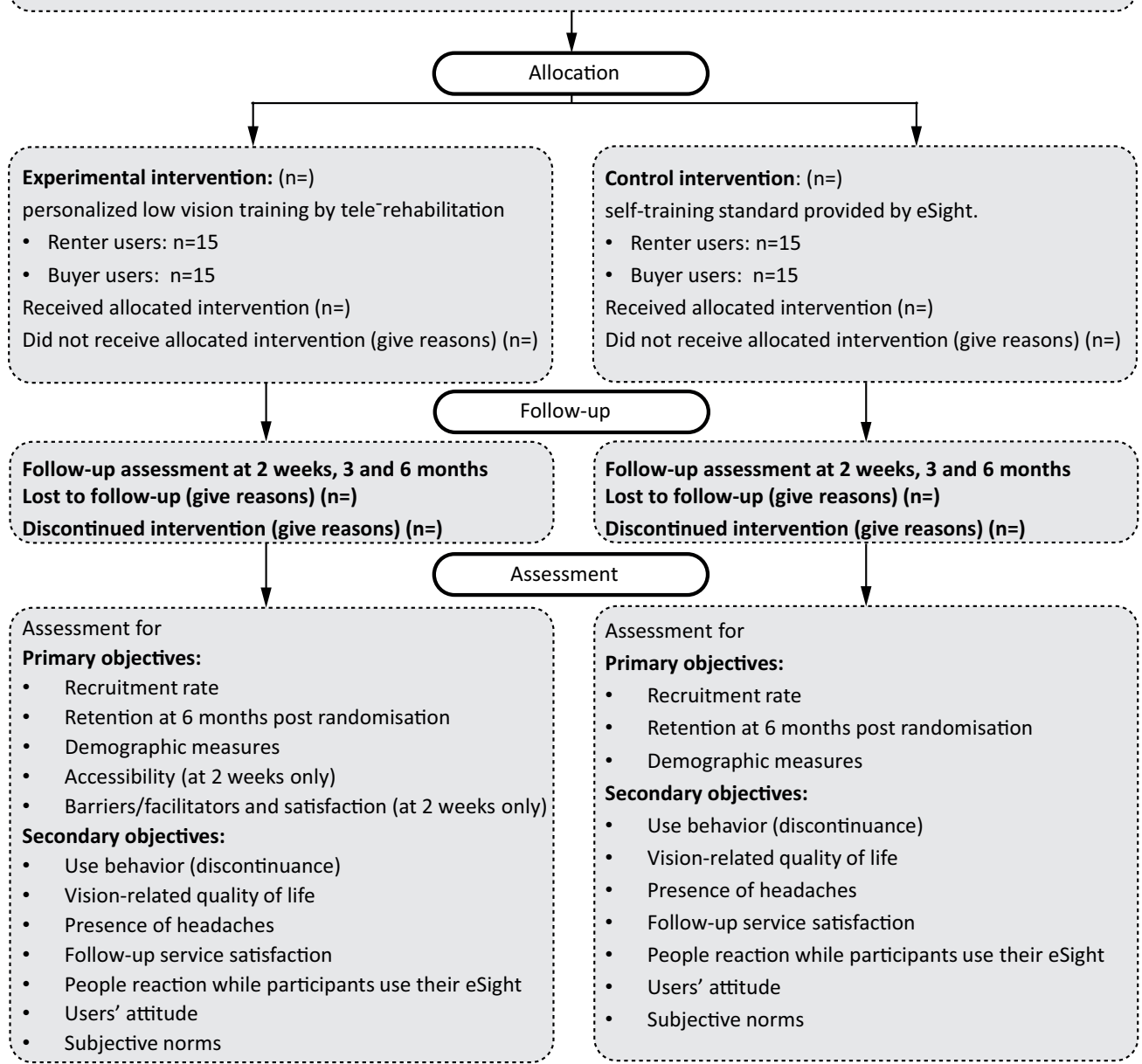

Figure 1 Flowchart showing planned participant flow. 
Table 1 The eSkills user guide, a self-training programme divided across 4 weeks

\begin{tabular}{ll}
\hline Week & Skills \\
\hline Week 1 & Learning the technical aspects of eSight Eyewear as well as the settings for distance vision and reading \\
Week 2 & $\begin{array}{l}\text { Exercises focused on distance vision and reading } \\
\text { Week } 3\end{array}$ \\
$\begin{array}{ll}\text { Refinement of the previous tasks associating additional complexity and introduces exercises for handwriting and } \\
\text { hand-eye coordination }\end{array}$ \\
Week 4 & $\begin{array}{l}\text { Improvement of the tasks involving hand-eye coordination and introducing viewing techniques on other media } \\
\text { (digital tablets, TV and so on) }\end{array}$ \\
\hline
\end{tabular}

will be determined by asking participants and the LV therapist independently about any problems related to internet connectivity, access to the videoconference platform, use of the hardware and audio/visual quality. At each training session, the LV therapist will report any problems in a diary, for each participant and herself, respectively. Regarding acceptability, all participants and the LV therapist will complete a satisfaction survey with a research assistant, containing quantitative and qualitative items, to indicate their preferences regarding the telerehabilitation intervention or the self-training programme, as well as to report barriers and facilitators. Participants will be asked to rate the experience of telerehabilitation or self-training for comfort, efficiency, effectiveness and likelihood for future use, rated on a 4-point Likert scale ('strongly agree' to 'strongly disagree'). The LV therapist will be asked to provide her overall perception and judgement of the telerehabilitation compared with her previous experience with face-to-face intervention (in-office sessions and/or home visits). In addition, we will ask participants for their written permission (consent form to be provided by mail) to contact their eye care professional to obtain an eye report, including visual information (ie, visual acuities, diagnosis).

\section{Exploratory/secondary outcomes}

In addition to the primary outcomes, we will collect measures about the impact of telerehabilitation on use behaviour (eSight Eyewear discontinuance rate) and on participants' quality of life (measured with two standardised device-related quality of life questionnaires, described in the Exploratory/secondary outcomes section). We will explore important predictors of eSight Eyewear use pre-identified in a cross-sectional study, ${ }^{16}$ such as the presence of headaches, follow-up service satisfaction, and other people's reaction while participants use their eSight device.

Discontinued device use will be monitored by follow-up evaluations and through questionnaires. In the context of our analyses, early discontinuance in device use is defined as a participant who stops using the device during the first 2 weeks of the study (either a renter who decides not to buy the device at the end of the rental period or a buyer who decides not to use it anymore), because this is the time period during which eSight Corporation offers a loan to customers. From a clinical research point of view, we will define discontinuance in device use as a participant reporting non-use of the device in the previous 3 months on any given task, as it has previously been applied in the context of LV devices in an outpatient population ${ }^{32}$ and as reported in a questionnaire specifically developed for this study, containing open and closed questions.

We will measure the impact of assistive technology on quality of life in a standardised and objective way with the Psychosocial Impact of Assistive Devices questionnaire (PIADS) ${ }^{33-35}$ and the Quebec User Evaluation of Satisfaction with Assistive Technology (QUEST), ${ }^{36} 37$ both of which are validated in English and French. The PIADS is a 26-item questionnaire originally developed to measure the effect of assistive devices on quality of life and was pilottested with eyeglasses and contact lenses. ${ }^{35}$ The PIADS has previously been used with LV magnification devices, specifically with CCTVs. ${ }^{38}{ }^{39}$ It is a reliable and valid tool and can predict assistive technology abandonment. ${ }^{33} 35$ There are three subscales: competence, adaptability and self-esteem. The QUEST) ${ }^{37}$ is a 12-item outcome measure that assesses user satisfaction with two components, device and services, with a wide range of assistive technology in a structured and standardised way. Psychometric properties have been tested with respect to test-retest stability, alternate-form equivalence, internal consistency and factorial composition validity, and appear to be adequate. ${ }^{37}$ Device dimension embraces eight items related to salient characteristics of the assistive technology whereas the services dimension encompasses four intercorrelated items.

To explore factors related to the use and discontinuance of use of the device in connection with using eSight, a questionnaire was specifically developed. It employs open and closed questions whose items refer to timing and frequency of use, nature of the task for which the device is used or discontinued. It is composed of 40 items, examining the use of eSight, user's characteristics, utilisation changes, social and physical environments and training/intervention.

\section{Participation timeline}

Each participant will be engaged in the study anywhere between 2 weeks (renters who choose not to purchase) and 6 months (buyers, and initial renter deciding to buy eSight Eyewear after the trial period) starting at randomisation, with assessments at baseline, 2 weeks, 3 and 6 months. After their recruitment, participants will be contacted by phone about an hour before the scheduled telerehabilitation session time, to guide them through 


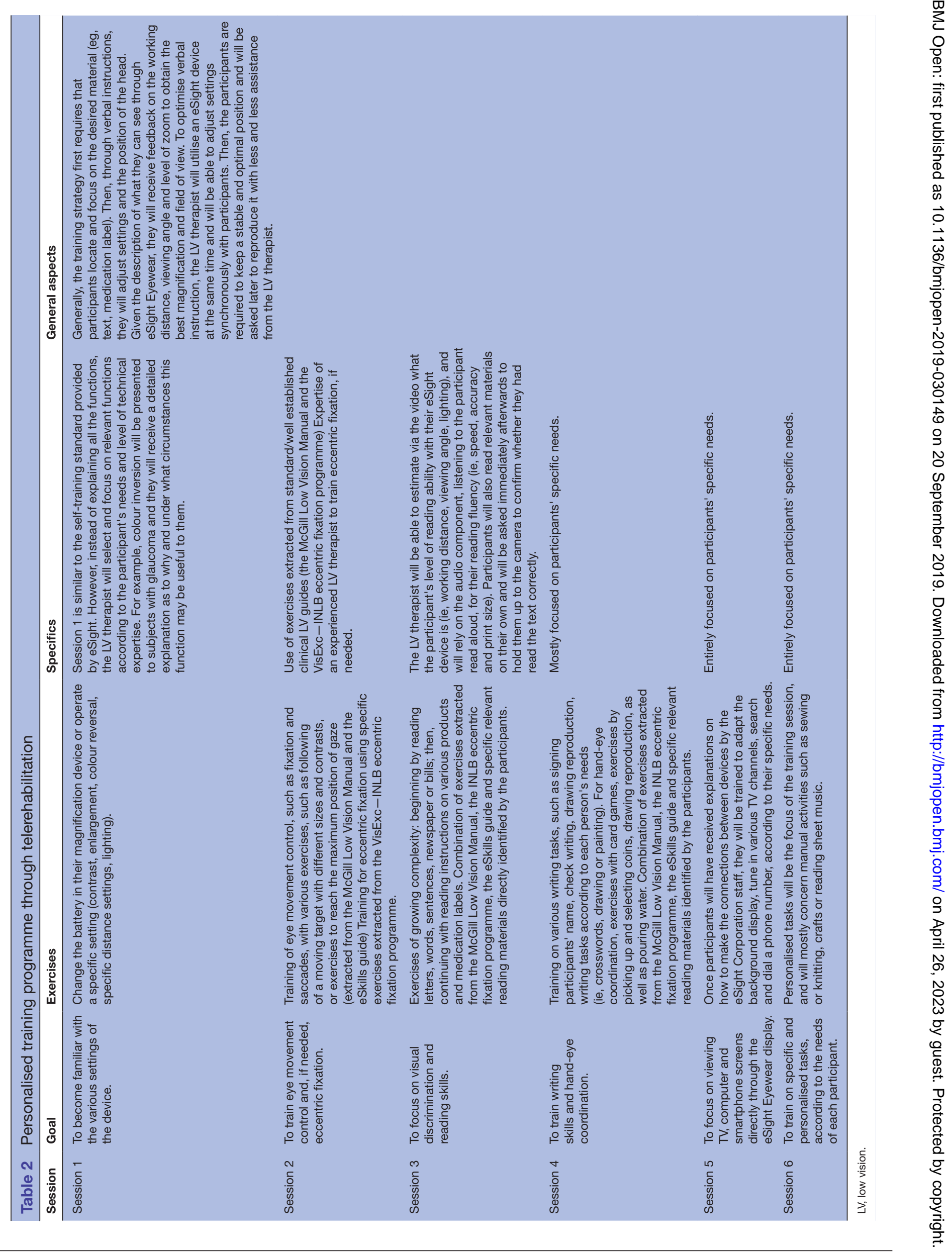


Table 3 Schedule of enrolment, interventions and assessments

\begin{tabular}{|c|c|c|c|c|c|}
\hline \multirow[b]{2}{*}{ Timepoint } & \multirow{2}{*}{$\begin{array}{l}\text { Enrolment } \\
0\end{array}$} & \multirow{2}{*}{$\begin{array}{l}\text { Allocation } \\
0\end{array}$} & \multicolumn{3}{|c|}{ Postallocation } \\
\hline & & & 2 weeks & 3 months & 6 months \\
\hline \multicolumn{6}{|l|}{ Enrolment } \\
\hline Informed consent & $x$ & & & & \\
\hline Demographic and clinical details & $x$ & & & & \\
\hline \multicolumn{6}{|l|}{ Interventions } \\
\hline Telerehabilitation & & $x$ & & & \\
\hline Self-training standard (eSkills User Guide) & & $\mathrm{x}$ & & & \\
\hline \multicolumn{6}{|l|}{ Assessment } \\
\hline \multicolumn{6}{|l|}{ Baseline } \\
\hline $\begin{array}{l}\text { Follow-up questionnaire (PIADS and QUEST; factors } \\
\text { related to the use of the device) }\end{array}$ & & & $x$ & $x$ & $\mathrm{x}$ \\
\hline \multicolumn{6}{|l|}{ Usage/discontinuance of eSight device } \\
\hline Accessibility of telerehabilitation* & & & $x$ & & \\
\hline Satisfaction with telerehabilitation* & & & $x$ & & \\
\hline Barriers and facilitators of telerehabilitation* & & & $x$ & & \\
\hline Lost to follow-up & & & $x$ & $x$ & $x$ \\
\hline Adverse outcomes & & & $x$ & $x$ & $\mathrm{x}$ \\
\hline
\end{tabular}

*Experimental group only.

PIADS, Psychosocial Impact of Assistive Devices questionnaire.

the setup process and help with any questions or issues related to accessing the videoconference portal. An initial questionnaire $(\sim 30 \mathrm{~min})$, administered by the $\mathrm{LV}$ therapist, will be used to collect data on demographics, health conditions and quality of life. Then, a second questionnaire $(\sim 45 \mathrm{~min})$, will be used for the three follow-up assessments. This questionnaire is composed of 94 questions adopted from the PIADS and the QUEST, and questions specifically developed for this study, and will be self-administered online, accessible through a URL link. The schedule of assessments is summarised in table 3 . This survey has been pilot-tested with four individuals, one with normal sight to confirm content consistency and general accessibility, followed by three individuals with visual impairment to confirm accessibility with technologies, two using ZoomText magnification software and one with JAWS screen reading software.

\section{Sample size}

A previous clinical trial using eSight Eyewear reported recruiting 74 individuals across 6 sites, of which 51 completed the study (drop-out rate of $31 \%$ ) ${ }^{13}$; however, a key difference was that the investigators provided eSight Eyewear as a loan for the period of the 3-month study, and participants were not self-selected device owners/renter, as is the case in the present study. Therefore, without direct comparison standard, we will recruit 60 participants in total, with 30 individuals in each group. This sample size is comparable to other feasibility studies in LV rehabilitation ${ }^{40}$ and is intended to maximise the available data given the opportunity created through the collaboration with the device manufacturer. Given the proposed recruitment period, this sample size will allow us to evaluate any possible limitations with enrolment and retention more robustly, and will provide rich data on accessibility and acceptability of a telerehabilitation training. A previous multicentre prospective study demonstrating the short and medium term effects of eSight Eyewear, reported various effect sizes that cover the secondary outcome measures proposed in the present protocol, ranging from $\omega^{2}=0.04$ to 0.83 . Choosing a conservative average effect size of $\omega^{2}=0.23$ to calculate the sample of the present study (based on a mixed design ANOVA), power analysis using $\mathrm{G}^{*}$ Power $^{41} 42$ indicated that, with a desired power of 0.95 and alpha level of 0.05 , the necessary sample size is $n=60$, with $n=30$ in each arm, a sample size commonly used in feasibility studies.

\section{Recruitment}

The projected study timeline is from September 2018 to December 2019. Directly after buying or renting an eSight device, clients will be informed by an employee 
of eSight Corporation of the opportunity of participate in the present research project. A letter introducing the study will be sent by email at the time of device delivery, and a paper version will be added in the parcel, providing the contact information of the research team to the users, so they can express their interest in participation. Once a person has expressed an interest, the LV therapist will provide verbal (by phone) and written (outline of the study and consent form will be sent by email to the potential participant) study information, explaining the objectives and the schedule of the study, will check eligibility and obtain informed written consent. Potential participants will be asked to sign, scan/photograph and return their consent form by email.

\section{Assignment of interventions}

Participants will be randomised to receive either personalised LV training by telerehabilitation, or the selftraining standard provided by eSight. Allocation will be at a 1:1 ratio, whereby two randomisations will be carried out separately at the Université de Montréal by a research assistant who is not involved in the study: one for the participants who bought the eSight Eyewear, the other for those who rented them. Given the small samples sizes, and in order not to take the risk of having unbalanced control and experimental groups, the first participant of each group will be allocated by coin toss, and then allocation of all following participants will alternate. The LV therapist is only notified of group allocation at the first testing session.

\section{Bias control}

Both participants and the LV therapist administering the protocol will be aware to which condition (control or experimental) the participants are allocated. Therefore, in order to minimise experimenter bias, we will use online follow-up questionnaires via an URL link (https:/ / www.hostedincanadasurveys.ca).

\section{Data collection, management and analysis}

Data collection and data management. Participants will be assigned a study number after consent prior to randomisation. Quantitative data will be tabulated using a Microsoft Office Excel spreadsheet. Interview data will be audio recorded and transcribed verbatim into a Microsoft Office Word document. All data will be analysed by the study principal investigator (M-CL), who will not meet the participants or be involved in data collection and will be masked to group membership until data collection is complete in order to reduce detection bias. Data will be stored centrally and kept in locked, secure access filing cabinets or on password-protected computers on Université de Montréal premises; this includes electronic data and case report forms and interview materials. These data will be stored for a period of 7 years until the end of the project, after which they will be destroyed.

\section{Statistical analyses}

Data will be analysed using JASP V.0.9 software..$^{43}$ The analysis plan consists of 3 steps. In step 1, descriptive statistics will be used to present the sample and responses on all outcome measures. In step 2, we will examine the primary outcome measures to evaluate feasibility, including recruitment, retention, accessibility and acceptability, while in step 3, we will explore possible effects within the secondary outcomes, including device abandonment and quality of life.

Step 1: descriptive measures, including participants' demographic and clinical characteristics, will be summarised as means and SD, medians and IQRs, and by counts and percentages as appropriate.

Step 2: enrolment will be analysed using the period of recruitment defined (from September 2018 to June 2019), reporting the number of participants assessed for eligibility and the number of participants who will be excluded (number of participants declining to participate and number of participants not meeting the inclusion criteria). We will report the number of participants who will be enrolled within the 10-month recruitment period and whether the minimum required $80 \% \quad(54$ participants) were reached.

Retention in both the experimental and the control groups will be analysed at 2 weeks, as well as 3 and 6 months using the number of participants remaining in the study. If participants wish to withdraw after they have been allocated to an intervention group, we will give them the opportunity to explain their reasoning, should they be interested in sharing these reasons and will record them in the case report.

Accessibility of telerehabilitation training (numbers and types of issues with: Internet connectivity, access to the videoconference platform, use of the hardware and audio/visual quality) will be presented as frequency counts as well as analysed using qualitative description. ${ }^{44}$

Acceptability will be measured using quantitative (satisfaction surveys) and qualitative measures (interview with the LV therapist). Quantitative measures (ordinal data) will be presented using descriptive statistics. Qualitative analysis will reveal barriers and facilitators related to telerehabilitation intervention. An interview with the LV therapist will be transcribed verbatim and coded by the first author and a research assistant, using a case study approach, starting with open coding. ${ }^{45}$ Significant sentences in each transcript that have relevance to the research question to help us better understand barriers and facilitators to the telerehabilitation intervention with eSight users, as experienced by the LV therapist, will be highlighted. ${ }^{46}$ Then, all codes will be grouped together according to common themes. Coding disagreements will be resolved in face-toface discussion, and any remaining disagreement will be decided by a third party (WW). Finally, the codes will be grouped into themes to establish an overall descriptive picture of the interview content that allows the reader to better understand the barriers and facilitators related to this telerehabilitation intervention. ${ }^{47}$ 
Step 3: secondary outcomes. (1) eSight Eyewear early discontinuance rate at 2 weeks and discontinuance rate at 6 months will be analysed using descriptive statistics. (2) Quality of life as measured by the PIADS and QUEST will be examined according to a factorial design approach (see figure 1), comparing outcomes for device renters with those of device owners; in addition, participants who are in the control group will be compared with those in the intervention group, both across follow-up time points (pre-intervention, after 2 weeks, as well after 3 and 6 months of device use). Given the repeated-measures component of the study, and in order to accommodate potentially missing data, the analyses will be conducted using a mixed-effects model, and post-hoc tests using Tukey's honestly significant difference correction. (3) Factors preidentified as being predictive of eSight, depending on the nature of the 40 items questionnaire, Spearman correlation coefficients and $\chi^{2}$ will be calculated to examine the relationships between ordinal (eg, quality of life score) and categorical (eg, discontinuation frequency) variables, respectively. Data collected up to the point of withdraw will be included in the data analysis.

\section{Study status}

Recruitment and data collection are currently ongoing. Recruitment is expected to be completed in June 2019, given minor delays in the timeline. Data collection is expected to be complete in December 2019, followed by data analyses and manuscript preparation in early 2020.

\section{Patient and public involvement}

The research questions and outcome measures of the present study were tailored to reflect the barriers and facilitators to eSight Eyewear use as identified by device users in a cross-sectional study. ${ }^{16}$ It is planned, once the telerehabilitation intervention is completed, to ask participants to assess the burden of the telerehabilitation intervention and the time required to participate, in order to design future interventions to accommodate accordingly.

\section{Limitations and possible solutions}

A limitation in our study is self-selection bias, as we will enrol participants who spontaneously decide to rent or purchase eSight Eyewear, express interest in research participation, own the computer equipment necessary for telerehabilitation and data collection, and are willing to try an Internet-based video conference platform. To overcome this potential bias, we plan to loan eSight Eyewear and tablets to participants in the next phase of this project.

Another limitation is that it will not always be possible to differentiate all possible reasons for discontinuance versus drop-out or other types of attrition. This will probably be the case specifically for participants lost to follow-up without explanation. However, through regular monitoring throughout the study, we hope to be able to record most reasons for leaving the study. Another potential limitation is the absence of an in-office evaluation with objective visual function measures, such as visual acuity, visual field or contrast sensitivity, which could provide complementary insight. Given the online survey nature of data collection, complete access to medical chart information will not be feasible. However, we will be able to obtain some objective information about visual function by asking participants to give the research team access to their most recent eye report.

The results of this study will be presented at local, national and international conferences that cater to LV rehabilitation professionals and researchers, and will be published in a peer-reviewed research journal. It is also planned to publish an article in a journal that is more focused on clinical outcomes in vision rehabilitation or on assistive technology.

Twitter Walter Wittich @walterwittich

Acknowledgements We thank Josée Duquette for her permission to use the VisEXc-INLB eccentric fixation programme.

Contributors WW and M-CL designed the study. WW secured funding. M-CL will conduct the study and will acquire the data. M-CL and WW will analyse the data. The present protocol was drafted by M-CL and critically reviewed and amended by WW.

Funding This study was funded by Mitacs Accelerate Fellowship IT08595 Grant. Competing interests None declared.

Patient consent for publication Obtained.

Ethics approval The protocol was reviewed and approved by the institutional review board of the Centre de recherche interdisciplinaire en réadaptation du Montréal métropolitain (CRIR\# 1286-1217).

Provenance and peer review Not commissioned; externally peer reviewed.

Open access This is an open access article distributed in accordance with the Creative Commons Attribution Non Commercial (CC BY-NC 4.0) license, which permits others to distribute, remix, adapt, build upon this work non-commercially, and license their derivative works on different terms, provided the original work is properly cited, appropriate credit is given, any changes made indicated, and the use is non-commercial. See: http://creativecommons.org/licenses/by-nc/4.0/.

\section{REFERENCES}

1. Foster A, Gilbert C, Johnson G. Changing patterns in global blindness: 1988-2008. Community Eye Health 2008;21:37-9.

2. Alabdulkader $B$, Leat SJ. Reading in children with low vision. $J$ Optom 2010;3:68-73.

3. Corn A, Lusk KE. Chapter 1: Perspectives on low vision. In: Corn A, Koenig A, eds. Foundations of low vision: clinical and functional perspectives. 2 edn. New York: AFB Press, 2010: 3-25.

4. Binns AM, Bunce C, Dickinson C, et al. How effective is low vision service provision? A systematic review. Surv Ophthalmol 2012;57:34-65.

5. Trauzettel-Klosinski S. Rehabilitation for visual disorders. J Neuroophthalmol 2010;30:73-84.

6. Wolffsohn JS, Peterson RC. A review of current knowledge on electronic vision enhancement systems for the visually impaired. Oph Phys Optics 2003;23:35-42.

7. Deemer AD, Bradley CK, Ross NC, et al. Low vision enhancement with head-mounted video display systems: are we there yet? Optom Vis Sci 2018;95:694-703.

8. Ortiz A, Chung ST, Legge GE, et al. Reading with a head-mounted video magnifier. Optom Vis Sci 1999;76:755-63.

9. Ballinger R, Lalle P, Maino J, et al. Veterans affairs multicenter low vision enhancement system (LVES) study: clinical results. Report 1: effects of manual-focus LVES on visual acuity and contrast sensitivity. Optometry 2000;71:764-74.

10. Geruschat DR, Deremeik JT, Whited SS. Head-mounted displays: are they practical for school-age children? J Vis Impair Blind 1999;93:485-97. 
11. Lin CS, Jan H-A, Lay Y-L, et al. Evaluating the image quality of closed circuit television magnification systems versus a headmounted display for people with low vision. Assist Technol 2014;26:202-8.

12. Culham LE, Chabra A, Rubin GS. Clinical performance of electronic, head-mounted, low-vision devices. Oph Phys Optics 2004:24:281-90.

13. Wittich W, Lorenzini M-C, Markowitz SN, et al. The effect of a head-mounted low vision device on visual function. Optom Vis Sci 2018;95:774-84.

14. Stelmack JA, Tang XC, Wei Y, et al. Outcomes of the veterans affairs low vision intervention trial II (LOVIT II): a randomized clinical trial. JAMA Ophthalmol 2017;135:96-104.

15. Lorenzini M-C, Wittich W. Factors related to the use of magnifying Iow vision AIDS: a scoping review. Disabil Rehabil 2019:1-13.

16. Lorenzini MC, Hamalainen A, Wittich W, eds. Factors related to the use of a head-mounted display for individuals with low vision. poster session presented at: from bench to bedside and back 91st ARVO annual meeting. Vancouver, Canada, 2019.

17. Pollard TL, Simpson JA, Lamoureux EL, et al. Barriers to accessing low vision services. Oph Phys Optics 2003;23:321-7.

18. Lam N, Leat SJ. Barriers to accessing low-vision care: the patient's perspective. Can J Ophthalmol 2013;48:458-62.

19. Goldstein JE, Massof RW, Deremeik JT, et al. Baseline traits of low vision patients served by private outpatient clinical centers in the United States. Arch Ophthalmol 2012;130:1028-37.

20. lezzoni LI, Killeen MB, O'Day BL. Rural residents with disabilities confront substantial barriers to obtaining primary care. Health Serv Res 2006;41:1258-75.

21. Gold D, Zuvela B, Hodge WG. Perspectives on low vision service in Canada: a pilot study. Can J Ophthalmol 2006;41:348-54

22. Brennan DM, Tindall L, Theodoros D, et al. A blueprint for telerehabilitation guidelines. Telemed J E Health 2010;2011:662-5.

23. Hailey D, Roine R, Ohinmaa A, et al. Evidence of benefit from telerehabilitation in routine care: a systematic review. $J$ Telemed Telecare 2011;17:281-7.

24. Crossland MD, Silva RS, Macedo AF. Smartphone, tablet computer and e-reader use by people with vision impairment. Ophthalmic Physiol Opt 2014;34:552-7.

25. Bittner AK, Wykstra SL, Yoshinaga PD, et al. Telerehabilitation for people with low vision. Cochrane Database Syst Rev 2014;2014. doi:10.1002/14651858.CD011019. [Epub ahead of print: 10 Mar 2014].

26. Bittner AK, Yoshinaga $P$, Bowers $A$, et al. Feasibility of telerehabilitation for low vision: satisfaction ratings by providers and patients. Optom Vis Sci 2018;95:865-72.

27. CONSORT group. Pilot and feasibility trials Ottawa, Canada: the CONSORT group, the Ottawa Hospital research Institute, 2018. Available: http://www.consort-statement.org/extensions/overview/ pilotandfeasibility [Accessed 8 May 2019].

28. eSight Corporation. eSkills User Guide \& Proficiency Program. Toronto, Canada: esight, 2015.

29. Williams J. The Declaration of Helsinki and public health. Bull World Health Organ 2008;86:650-1.
30. Duquette J, Lapointe N, Loiselle J. VisExc - INLB : méthode d'évaluation et d'entraînement la vision excentrique de l'Institut Nazareth et Louis-Braille : manuel de I'usager. Institut Nazareth et Louis-Braille, 2013.

31. Overbury O, Conrod EB. McGill low vision manual. Betacom Group, 1997.

32. Dougherty BE, Kehler KB, Jamara R, et al. Abandonment of low-vision devices in an outpatient population. Optom Vis Sci 2011;88:1283-7.

33. Day H, Jutai J, Campbell KA. Development of a scale to measure the psychosocial impact of assistive devices: lessons learned and the road ahead. Disabil Rehabil 2002;24:31-7.

34. Demers L, Monette M, Descent M, et al. The psychosocial impact of assistive devices scale (PIADS): translation and preliminary psychometric evaluation of a Canadian-French version. Qual Life Res 2002;11:583-92.

35. Day $\mathrm{H}$, Jutai J. Measuring the psychosocial impact of assistive devices: the PIADS. Can J Rehabil 1996;9:159-68.

36. Demers L, Weiss-Lambrou R, Ska B. Development of the Quebec user evaluation of satisfaction with assistive technology (QUEST). Assist Technol 1996;8:3-13.

37. Demers L, Weiss-Lambrou R, Ska B. The Quebec user evaluation of satisfaction with assistive technology (QUEST 2.0): an overview and recent progress. Technol Disabil 2002;14:101-5.

38. Strong G, Jutai JW, Bevers P, et al. The psychosocial impact of closed-circuit television (CCTV) low vision AIDS. Vis Impair Res 2003;5:179-90.

39. Huber JG, Jutai JW, Strong JG, et al. Psychosocial impact of closed-circuit television (CCTV) devices in age-related macular degeneration. J Vis Impair Blind 2008;108:690-701.

40. Crossland MD, Thomas R, Unwin $\mathrm{H}$, et al. Tablet computers versus optical AIDS to support education and learning in children and young people with low vision: protocol for a pilot randomised controlled trial, CREATE (Children Reading with Electronic Assistance To Educate). BMJ Open 2017;7:e015939.

41. Faul F, Erdfelder E, Lang A-G, et al. G*Power 3: a flexible statistical power analysis program for the social, behavioral, and biomedical sciences. Behav Res Methods 2007;39:175-91.

42. Faul F, Erdfelder E, Buchner A, et al. Statistical power analyses using $\mathrm{G}^{\star}$ Power 3.1: tests for correlation and regression analyses. Behav Res Methods 2009;41:1149-60.

43. JASP [computer program]. Version 0.9. Amsterdam: University of Amsterdam, 2017.

44. Sandelowski M. Whatever happened to qualitative description? Res Nurs Health 2000;23:334-40.

45. Elo $\mathrm{S}$, Kyngäs $\mathrm{H}$. The qualitative content analysis process. J Adv Nurs 2008:62-107.

46. Creswell JW. Qualitative inquiry \& research design : choosing among five approaches. 2 edn. Thousand: Sage Publications, 2007.

47. Colorado State University. Conducting qualitative \& quantitative research : qualitative research methods, 2016. Available: https:// writing.colostate.edu/guides/index.cfm?categoryid=18\&title=4 [Accessed 10 Jan 2019]. 